\title{
Early Surfactant Therapy With Nasal Continuous Positive Airway Pressure or Continued Mechanical Ventilation in Very Low Birth Weight Neonates With Respiratory Distress Syndrome
}

\author{
Bita Najafian ${ }^{1,2}$; Seyed Hasan Fakhraie ${ }^{2}$; Seyed Abulfazl Afjeh ${ }^{2, *}$; Mohammad Kazemian ${ }^{2}$; \\ Majid Shohrati ${ }^{3}$; Amin Saburi ${ }^{3}$ \\ ${ }^{1}$ Department of Pediatrics, Faculty of Medicine, Baqiyatallah University of Medical Sciences, Tehran, IR Iran \\ ${ }^{2}$ Department of Pediatrics, Faculty of Medicine, Shahid Beheshti University of Medical Sciences, Tehran, IR Iran \\ ${ }^{3}$ Chemical Injuries Research Center, Baqiyatallah University of Medical Sciences, Tehran, IR Iran \\ *Corresponding Author: Seyed Abulfazl Afjeh, Department of Pediatrics, Faculty of Medicine, Shahid Beheshti University of Medical Sciences, Tehran, IR Iran. Tel/Fax:+98-2188600067, \\ E-mail: abolfazlafjeh@gmail.com; md.researcher@yahoo.com
}

Received: May 14, 2013; Revised: June 9, 2013; Accepted: June 29, 2013

\begin{abstract}
Background:Various strategies have been suggested for the treatment of respiratory distress syndrome (RDS).
Objectives: The aim of this study was to compare the efficacies of two common methods of RDS management among neonates with low birth weight.

Patients and Methods: A cohort study was conducted on 98 neonates with definite diagnosis of RDS during 2008-2009. The neonates were divided into two groups by a blinded supervisor using simple randomization (odd and even numbers). Forty-five cases in the first group were treated with intubation, surfactant therapy, extubation (INSURE method) followed by nasal continuous positive airway pressure (N.CPAP) and 53 cases in the second group underwent intubation, surfactant therapy followed by mechanical ventilation (MV). Results: Five (11.1\%) cases in the first group and $23(43 \%)$ cases in the second group expired during the study. The rates of MV dependency among cases with INSURE failure and cases in the MV group were $37 \%$ and $83 \%$, respectively $(\mathrm{P}<0.001)$. Birth weight $(\mathrm{BW})(\mathrm{P}=0.017)$, presence of retinopathy of prematurity $(\mathrm{P}=0.022), \mathrm{C} / \mathrm{S}$ delivery $(\mathrm{P}=0.029)$ and presence of lung bleeding $(\mathrm{P}=0.010)$ could significantly predict mortality in the second group, although only $\mathrm{BW}(\mathrm{P}=0.029)$ had a significant impact on the mortality rate in the first group. Moreover, $\mathrm{BW}$ was significantly related to the success rate in the first group $(\mathrm{P}=0.001)$.

Conclusions: Our findings demonstrated that INSURE plus NCPAP was more effective than the routine method (permanent intubation after surfactant prescription). In addition, the lower rates of mortality, MV dependency, duration of hospitalization, and complications were observed in cases treated with the INSURE method compared to the routine one.
\end{abstract}

Keywords:Respiration, Artificial; Infant, Newborn; Respiratory Distress Syndrome, Newborn

\section{Background}

Mortality and morbidity rate in children with very low birth weight (VLBW) and prematurity remains high in spite of developments in therapeutics and instrumentation especially during the recent years (1). Respiratory distress syndrome (RDS) is one of the most common and fatal complications in premature neonates. Definite pathogenesis of this disease is the deficiency of surfactant due to prematurity of alveolar cells. Therefore, exogenous surfactant therapy is the main treatment $(2,3)$. A major issue in surfactant therapy among premature neonates is the time and method of prescription. Surfactant is usually prescribed via naso/oro tracheal tube, while it is necessary for neonates to be intubated for this method
$(3,4)$. On the other hand, orotracheal intubation is one of the important risk factors for pneumonia, bronchopulmonary dysplasia (BPD) and it can increase the duration of hospitalization (5). In addition to pneumothorax, bronchopleural fistula, and development of nosocomial pneumonia, and ventilator dependency were reported in neonates intubated for long time (6).

Various protocols have been proposed to decrease the duration of intubation. One is the INSURE protocol which includes intubation, surfactant prescription, and extubation followed by nasal continuous positive airway pressure (NCPAP) (7). This protocol makes these neonates less exposed to the complications of intubation versus

Implication for health policy/practice/research/medical education:

Respiratory Distress Syndrome is a common but life-threatening condition in preterm neonates and surfactant therapy is the main treatment for them. There are many protocols available for the treatment of these children by surfactant, but it remains as a challenging issue in neonatal intensive care units we focused on in this study.

Copyright @ 2014, Iranian Red Crescent Medical Journal; Published by Kowsar Corp. This is an open-access article distributed under the terms of the Creative Commons Attribution License, which permits unrestricted use, distribution, and reproduction in any medium, provided the original work is properly cited. 
routine protocol including intubation, surfactant prescription, and mechanical ventilation (MV) (8). Moreover, there are a few reports that confirmed the efficacy of early treatment with surfactant and early use of NCPAP to reduce the need for intubation and continues MV and its complications (9). In addition, there are some evidence indicating the benefit of MV for neonate with irregular and non-spontaneous respiration, also the efficacy of CPAP in these patients has been reported (10). Previous studies showed the advantages of both protocols to reduce mortality and morbidity of children with RDS (11-13), but there are some queries on the efficacy of these protocols in children with RDS and VLBW. It seems that more clinical studies are required to compare the efficacy of the two mentioned protocols.

\section{Objectives}

The aim of this study was to compare the efficacy of these two methods (INSURE plus NCPAP versus intubation, surfactant therapy and maintained MV) for the treatment of VLBW neonates with RDS.

\section{Patients and Methods}

\subsection{Study Population}

A cohort study was conducted on neonates born between 2008 and 2009 in a tertiary care hospital (Mofid Hospital) in Tehran, Iran. Newborns with clinical symptoms of RDS and birth weights less than $1500 \mathrm{~g}$ (VLBW) were consecutively included. RDS was diagnosed by one neonatologist according to clinical symptoms (cyanosis, grunting, retractions, and tachypnea), radiographic studies (ground glass or air bronchograms pattern in CXR) and physical examination (14). Patients with congenital anomalies, pulmonary structure abnormalities, chromosomal abnormalities, any evidence of sepsis (maternal and neonatal confirmed by positive blood culture) and congenital heart diseases were excluded. Among 893 cases, finally, 98 neonates with RDS score more than 6, i.e. moderate to severe degree (Table 1 ), were included (15). The two groups were matched regarding the severity of RDS, maternal situations, nutritional status of the parturient, indications for LSCS, lack of evidence for meconium aspiration and other confounding variables as seen in Table 2 and presented P values.

\begin{tabular}{llll}
\hline Table 1. RDS Scoring $(15)$ & & & \\
\hline Score & $\mathbf{0}$ & $\mathbf{1}$ & $\mathbf{2}$ \\
\hline Respiration rate, breath/min & $\leq 60$ & $60-80$ & $\geq 80$ \\
FIO $_{\mathbf{2}}$ & $>40 \%$ & $21-40 \%$ & $<21 \%$ \\
Retraction & & & mild \\
$\quad$ Intercostals & None & Moderate to severe & mild \\
$\quad$ Sub costal & None & Moderate to severe & Audible without stethoscope \\
\hline Grunting & None & Audible with stethoscope & \\
\hline
\end{tabular}

\begin{tabular}{llll}
\hline \multicolumn{2}{l}{ Table 2. Baseline Characteristics $\mathrm{a}, \mathrm{b}, \mathrm{c}, \mathrm{d}$} & & \\
\hline Variable & First Group & Second Group & P Value \\
\hline Gender & $25(46.3)$ & $29(53.7)$ & 0.934 \\
\hline Male & $20(45.5)$ & $24(54.5)$ & \\
\hline Female & & & 0.054 \\
\hline Delivery & $6(24)$ & $19(76)$ & \\
\hline NVD & $39(53.4)$ & $34(46.6)$ & 0.211 \\
\hline C/S & & & \\
\hline Parity & $31(50.8)$ & $30(49.2)$ & \\
\hline Primiparity & $14(37.8)$ & $23(62.2)$ & 0.744 \\
\hline Multiparity & $1171 \pm 218$ & $1157 \pm 210$ & 0.140 \\
\hline Birth Weight, g & $29.60 \pm 2.28$ & $28.94 \pm 2.08$ & \\
\hline Gestational Age, wk &
\end{tabular}

\footnotetext{
a Abbreviations: C/S, cesarean section; NVD, normal vaginal delivery.

b First group underwent INSURE and then NCPAP.

c Second group underwent intubation, surfactant therapy and then MV.

$\mathrm{d}$ Data are presented as No. (\%).
} 


\subsection{Intervention}

To prevent the selection bias, the patients were divided into two groups by one supervisor using simple randomization method (odd and even numbers); newborns in the first group underwent INSURE and then NCPAP (with $4 \mathrm{~cm} / \mathrm{H}_{2} \mathrm{O}$ ), and those in the second group underwent intubation, surfactant therapy and then MV. One-hundred $\mathrm{mg} / \mathrm{kg}$ surfactant (Intratracheal Suspension of Survanta, Beractant, Columbus, Ohio, USA) warmed to $37^{\circ} \mathrm{C}$ was prescribed at each injection. At every time after the treatment, patients in the first group that their RDS symptoms were resolved or had a suitable arterial blood gas (ABG) analysis and also acceptable control chest X-Ray were considered as successful INSURE. If their symptoms remained stable with $\mathrm{FiO}_{2}<40$, positive end expiratory pressure $(\mathrm{PEEP})<5 \mathrm{~cm} / \mathrm{H}_{2} \mathrm{O}$ and in $\mathrm{ABG}$ analysis; $\mathrm{PaCO}_{2}$ $<60 \mathrm{mmHg}, \mathrm{PaO}_{2}>50 \mathrm{mmHg}$ and $\mathrm{PH}>7.25$, they were considered stable and their treatment were continued by oxygen-hood and followed up. In contrast, patients in the first group who had $\mathrm{O}_{2}$ saturation under $85 \%$, and in $\mathrm{ABG}$ analysis as $\mathrm{PaCO}_{2}>60 \mathrm{mmHg}, \mathrm{PaO}_{2}<50 \mathrm{mmHg}$ and $\mathrm{PH}<$ 7.2 while the PEEP increased to $6 \mathrm{~cm} \mathrm{H}_{2} \mathrm{O}$ underwent intubation and MV and they entitled as INSURE failure group (16). Additional dose of surfactant was prescribed when $\mathrm{FiO}_{2}$ was more than 40 and required $\mathrm{O}_{2}$ saturation to increase to $85 \%$. The study protocol was shown in Figure 1.

\subsection{Statistical Analysis}

Quantitative data were extracted as mean \pm standard deviation (SD). Statistical analyses were performed by using SPSS software (16th edition, SPSS Inc., Chicago). Normal distribution of data was checked by using Kolmogorov-Smirnov test, and those variables without

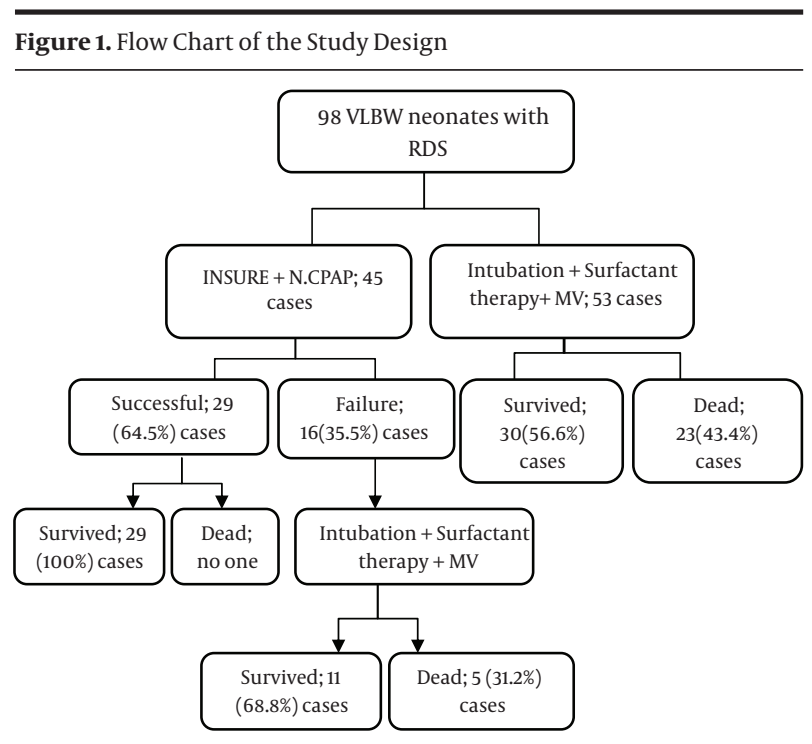

RDS; Respiratory distress syndrome, MV; mechanical ventilation, INSURE; intubation plus surfactant therapy and extubation, VLBW; very low birth weight, N.CPAP; Nasal Continuous Positive Airway Pressure. normal distribution were tested by nonparametric method. Independent t-test, Mann-Whitney U test and ANOVA (for two or more independent groups) were used for analyses. Variables with P-value less than 0.2 in univariate analysis were entered the logistic regression model. P-value $<0.05$ was considered statistically significant. In addition, statistician was blind to the groups. Dependent variables such as duration of hospitalization, mortality rate, complications of treatment including pneumothorax and ventilator dependency were considered as the efficacy of treatment. The intervention began after obtaining informed consent signed by the parents. There was no extra charge imposed to the patients' parents. The study protocol and proposal were approved by the Ethics Committee of Medical School of Shahid Beheshti University of Medical Sciences at 2009 (approval code: No.221). Parents of neonates could leave the study at any time. Data was inserted in checklist using blind codes by keeping the privacy of patients.

\section{Results}

Ninety-eight neonates (45 in the first group; INSURE+NCPAP and 53 in the second group (intubation and MV)) completed the study. The overall mean \pm SD of gestational age was $29.24 \pm 2.19$ weeks and the mean birth weight was $1.164 \pm 2.13 \mathrm{~g}$. In general, 54 (55.1\%) cases were male, 73 (74.4\%) neonates were born via elective cesarean section (C/S) and $61(62.2 \%)$ mothers were primiparous. Twenty-five cases (55.5\%) in the first group were male compared to 29 (54.7\%) in the second group. In addition, six cases (13.3\%) in the first group were born via NVD compared to 19 (35.8\%) in the second group. The two groups were matched regarding demographic and clinical characteristics (Table 2). A single dose of antenatal corticosteroids was administered for all cases. The most common gestational complication was gestational hypertension (19.1\%) followed by prolonged rupture of membrane (4\%) and placenta abruption (2\%). The mean Apgar score between the two groups was not statistically different $(\mathrm{P}=$ 0.172 ).

There was no significant difference in gestational age $(\mathrm{P}$ $=0.140)$, birth weight $(P=0.740)$, gender $(P=0.934)$ and time of surfactant prescription $(\mathrm{P}=0.521)$ between the two groups (Table 2). The success rate in INSURE group was $64.4 \%$ (29 cases), while condition of 16 (35.6\%) cases became worse and they underwent MV after INSURE failure. In MV group, 30 cases (56\%) survived at the end of study, while $5(11.1 \%)$ cases in the first group and $23(43 \%)$ cases of the second group died during the study. The most common cause of death was respiratory failure (46.42\%), followed by sepsis (42.85\%) and NEC (10.71\%). INSURE method in all expired newborns of the first group was failed and they underwent respiratory support by MV (Figure 1). A significant difference was found between the two subgroups (successful and failure) among the patients of the first group (INSURE method) regarding birth weight $(\mathrm{P}<$ 
0.001, $\mathrm{r}=-5.20)$, gestational age (GA) (P: 0.001, $\mathrm{r}=-3.655)$, N.CPAP duration $(\mathrm{P}<0.001, \mathrm{~F}=18.531)$, lung bleeding ( $\mathrm{P}$ $<0.001$, odds ratio (OR): 0.237 , confidence interval (CI) [95\%]: 0.134-0.419), presence of necrotizing enterocolitis (NEC) ( $\mathrm{P}=0.012$, OR:0.293, CI [95\%]: 0.182-0.471) and mortality (P = 0.004, OR:0.275, CI[95\%]: 0.166-0.455) (Table 3). GA was the only variable associated with duration of hospitalization between the two subgroups, successful and unsuccessful INSURE $(\mathrm{P}=0.001)$. Moreover, $\mathrm{GA}(\mathrm{P}<0.001$, $\mathrm{r}=3.669)$, birth weight $(\mathrm{P}<0.001, \mathrm{r}=4.358)$, delivery via $\mathrm{C} / \mathrm{S}(\mathrm{P}=0.048$, OR: 1.889, CI [95\%]:1.029-3.468), lung bleeding ( $\mathrm{P}<0.001$, OR: 6.923, CI [95\%]: 2.547-18.817) and presence of NEC $(\mathrm{P}=0.015, \mathrm{OR}: 4.333, \mathrm{CI}[95 \%]$ : 1.243-15.104) were significantly different between neonates expired or survived (Table 4). In the INSURE group, prevalence of pneumothorax ( $\mathrm{P}=0.033$, OR: 0.178, CI [95\%]: 0.037-0.750), lung bleeding ( $\mathrm{P}=0.037$, OR: 0.358, CI [95\%]: 0.134-0.961), mortality (P<0.001, OR: 0.163, CI [95\%]: 0.056-0.479) and duration of need to $\mathrm{O}_{2}$ support $(\mathrm{P}=0.018, \mathrm{r}=-2.399, \mathrm{CI}[95 \%]$ : -13.24-1.25) were significantly lower than the MV group, and there were no significant differences for other items between the two groups ( $\mathrm{P}>0.05)$.

Variables with $\mathrm{P}<0.2$ in univariate analysis were included for multivariate analysis. Logistic regression test indicated that only birth weight $(\mathrm{P}=0.001, \mathrm{EXP}[\beta]$ : 0.009, CI [95\%]: 1.003-0.014) had a significant association with success rate in the first group. In addition, birth weight $(P=0.017, \operatorname{EXP}[\beta]: 0.995, C I[95 \%]: 0.991-0.999)$, presence of retinopathy of prematurity $(\mathrm{ROP})(\mathrm{P}=0.022, \mathrm{EXP}[\beta]$ : 0.011, $\mathrm{CI}[95 \%]$ : 0.000-0.516), delivery via $\mathrm{C} / \mathrm{S}(\mathrm{P}=0.029$, EXP $[\beta]$ : 0.129 , CI [95\%]: 0.021-0.810) and presence of lung bleeding $(\mathrm{P}=0.010, \operatorname{EXP}[\beta]: 14.220, \mathrm{CI}[95 \%]$ : 1.862-18.603) could significantly predict death in the second group, although only birth weight $(\mathrm{P}=0.029, \mathrm{EXP}[\beta]$ : 0.993, CI [95\%]: 0.9870.999 ) had a significant influence on the mortality rate in the first group. The incidence of MV dependency (that was defined at least more than 2 days) in INSURE failure subgroup compared to the MV group was $37 \%$ and $83 \%$, respectively, which indicated a statistically significant difference $(\mathrm{P}<0.001)$.

\begin{tabular}{|c|c|c|c|c|c|}
\hline Variable & Successful & Failure & PValue & Duration of Hospitalization & P Value \\
\hline Delivery & & & & & 0.960 \\
\hline NVD & $4(66.7)$ & $2(33.3)$ & 0.995 & 31.8 & \\
\hline $\mathrm{C} / \mathrm{S}$ & $25(64.1)$ & $14(35.9)$ & & 38.3 & \\
\hline Gravidity & & & & & 0.227 \\
\hline Primiparity & $22(71)$ & $9(29)$ & 0.639 & 35 & \\
\hline Multiparity & $7(50)$ & $7(50)$ & & 42 & \\
\hline Gender & & & & & 0.166 \\
\hline Male & $17(68)$ & $8(32)$ & 0.362 & 33.8 & \\
\hline Female & $12(60)$ & $8(40)$ & & 42.1 & \\
\hline Twins & & & & & 0.897 \\
\hline Singleton & $12(52.2)$ & $11(47.8)$ & $>0.999$ & 33.8 & \\
\hline Twin & $17(77.3)$ & $5(22.7)$ & & 37 & \\
\hline G-HTN & & & & & 0.829 \\
\hline Yes & $4(57.1)$ & $3(42.9)$ & 0.423 & 37 & \\
\hline No & $25(65.5)$ & $13(34.2)$ & & 39 & \\
\hline Birth weight, $g$ & & & & & 0.164 \\
\hline$<1000$ & $1(11.1)$ & $8(88.9)$ & $0.002^{\mathrm{C}}$ & 46.6 & \\
\hline $1000-1250$ & $12(70.6)$ & $5(29.4)$ & & 39 & \\
\hline $1250-1500$ & $16(84.2)$ & $3(15.8)$ & & 31.7 & \\
\hline Gestational age, wk & & & & & 0.001 \\
\hline
\end{tabular}


Najafian B et al.

\begin{tabular}{|c|c|c|c|c|c|}
\hline$<28$ & $5(33.3)$ & $10(66.7)$ & 0.465 & 51.5 & \\
\hline $28-32$ & $20(76.9)$ & $6(23.1)$ & & 31.9 & \\
\hline 33-37 & $4(100)$ & $0(0)$ & & 20.7 & \\
\hline Surfactant Dose & & & & & 0.756 \\
\hline Single & $28(68.3)$ & $13(31.7)$ & $>0.999$ & 37 & \\
\hline Multiple & $1(25)$ & $3(75)$ & & 34 & \\
\hline Time of surfactant administration, hr & & & & & 0.256 \\
\hline$<2$ & $8(72.7)$ & $3(27.3)$ & 0.586 & 29 & \\
\hline $2-4$ & $12(66.7)$ & $6(33.3)$ & & 41 & \\
\hline $4-6$ & $14(44.4)$ & $5(55.6)$ & & 46 & \\
\hline $7-12$ & $1(49.4)$ & $1(50.6)$ & & 25 & \\
\hline $13-24$ & $4(80)$ & $1(20)$ & & 30 & \\
\hline Duration of N.CPAP, hr & & & & & 0.195 \\
\hline$<6$ & $8(100)$ & $0(0)$ & 0.586 & 26 & \\
\hline $7-12$ & $3(75)$ & $1(25)$ & & 38 & \\
\hline $13-24$ & $8(88.9)$ & $1(11.1)$ & & 35 & \\
\hline $24-48$ & $7(63.6)$ & $4(36.4)$ & & 34 & \\
\hline$>48$ & $4(23.1)$ & $1(76.9)$ & & 47 & \\
\hline Pneumothorax & & & & & 0.919 \\
\hline Yes & $0(0)$ & $2(100)$ & 0.212 & 37 & \\
\hline no & $29(67.4)$ & $14(32.6)$ & & 37 & \\
\hline Sepsis & & & & & 0.641 \\
\hline Yes & $6(60)$ & $4(40)$ & $>0.999$ & 37 & \\
\hline No & $23(65.7)$ & $12(34.3)$ & & 33 & \\
\hline NEC & & & & & 0.734 \\
\hline Yes & $0(0)$ & $4(100)$ & 0.555 & 37 & \\
\hline No & $29(70.7)$ & $12(29.3)$ & & 33 & \\
\hline ROP & & & & & 0.734 \\
\hline Yes & $2(66.7)$ & $14(33.3)$ & 0.304 & 37 & \\
\hline No & $8(33.3)$ & $2(66.7)$ & & 33 & \\
\hline Lung bleeding & & & & & 0.735 \\
\hline Yes & $6(60)$ & $4(40)$ & 0.210 & 37 & \\
\hline No & $23(65.7)$ & $12(34.3)$ & & 39 & \\
\hline
\end{tabular}

a Abbreviations: C/S, cesarean section; G-HTN, gestational hypertension; N.CPAP, nasal continuous positive airway pressure; NEC, necrotizing enterocolitis; NVD, normal vaginal delivery; ROP, retinopathy of prematurity.

$\mathrm{b}$ Data are presented as No.(\%).

c Statistically significant. 
Najafian B et al.

\begin{tabular}{|c|c|c|c|c|c|}
\hline Variable & Survived & Dead & P Value & Duration of Hospitalization & PValue \\
\hline Delivery & & & 0.111 & & 0.554 \\
\hline NVD & $8(42.1)$ & $11(57.9)$ & & 40.3 & \\
\hline $\mathrm{C} / \mathrm{S}$ & $22(64.7)$ & $12(35.3)$ & & 35.9 & \\
\hline Gravidity & & & 0.960 & & 0.147 \\
\hline Primiparity & $14(46.7)$ & $16(53.3)$ & & 33 & \\
\hline Multiparity & $16(69.6)$ & $7(30.4)$ & & 43.3 & \\
\hline Gender & & & 0.745 & & 0.381 \\
\hline Male & $17(58.6)$ & $12(41.4)$ & & 34.6 & \\
\hline Female & $13(54.2)$ & $11(45.8)$ & & 40.9 & \\
\hline Twins & & & 0.413 & & 0.456 \\
\hline Singleton & $19(52.8)$ & $17(47.2)$ & & 35.6 & \\
\hline Twin & $11(46.7)$ & $6(35.3)$ & & 41.3 & \\
\hline Birth weight, gr & & & $0.002^{\mathrm{C}}$ & & 0.576 \\
\hline$<1000$ & $4(28.6)$ & $10(71.4)$ & & 33.5 & \\
\hline $1000-1250$ & $10(47.6)$ & $11(52.4)$ & & 42 & \\
\hline $1250-1500$ & $16(88.9)$ & $2(11.1)$ & & 35.2 & \\
\hline Gestational age, wk & & & $<0.001$ & & 0.335 \\
\hline$<28$ & $7(29.2)$ & $17(70.8)$ & & 39.6 & \\
\hline $28-32$ & $22(84.6)$ & $4(15.4)$ & & 37.9 & \\
\hline $33-37$ & $1(33.3)$ & $2(66.7)$ & & 16.3 & \\
\hline Surfactant Dose & & & 0.177 & & 0.186 \\
\hline Single & $25(61)$ & $16(39)$ & & 40 & \\
\hline Multiple & $5(36)$ & $7(64)$ & & 28 & \\
\hline Time of surfactant administration, hr & & & 0.850 & & 0.724 \\
\hline$<2$ & $14(58.3)$ & $10(41.7)$ & & 41 & \\
\hline $2-4$ & $6(60)$ & $4(40)$ & & 37 & \\
\hline $4-6$ & $4(66.7)$ & $2(33.3)$ & & 41 & \\
\hline $7-12$ & $5(45.5)$ & $6(54.5)$ & & 29 & \\
\hline $13-24$ & $1(100)$ & $0(0)$ & & 37 & \\
\hline Pneumothorax & & & & & 0.303 \\
\hline Yes & $6(54.5)$ & $5(45.5)$ & $>0.999$ & 35 & \\
\hline no & $24(57.1)$ & $18(42.9)$ & & 44 & \\
\hline Sepsis & & & 0.194 & & 0.722 \\
\hline Yes & $13(68.4)$ & $6(31.6)$ & & 38 & \\
\hline No & $17(50)$ & $17(50)$ & & 35 & \\
\hline NEC & & & 0.272 & & 0.616 \\
\hline Yes & $3(37.5)$ & $5(62.5)$ & & 38 & \\
\hline No & $27(60)$ & $18(40)$ & & 33 & \\
\hline ROP & & & 0.100 & & 0.001 \\
\hline Yes & $2(66.7)$ & $1(33.3)$ & & 82 & \\
\hline No & $28(56)$ & $22(44)$ & & 34 & \\
\hline Lung bleeding & & & 0.014 & & 0.475 \\
\hline Yes & $6(43.3)$ & $12(66.7)$ & & 39 & \\
\hline No & $24(68.6)$ & $11(31.4)$ & & 33 & \\
\hline
\end{tabular}




\section{Discussion}

Our findings showed that INSURE method with N.CPAP was safer and more effective than the routine method (Intubation plus MV after surfactant therapy) in VLBW children with RDS. Additionally, INSURE method was associated with lower mortality, morbidity rate and less dependency to MV during hospitalization, and the mentioned factors could be considered as success (efficacy) criteria compared with the previous study regarding efficacy and MV dependency.

Previous reports compared the efficacy and safety of various methods of RDS management. There is a controversy to choose the best method for pulmonary support of neonates with VLBW and RDS. However, the efficacy of N.CPAP compared to permanent MV has been reported in previous studies but it is not clear when and how it should be used (13, 17-21).

Nowadays, positive airway pressure including N.CPAP has been used for the treatment of neonates with RDS after surfactant therapy. Ancora et al. investigated the efficacy of bi-level positive airway pressure (Bi-PAP) for neonates after INSURE failure. They found that MV dependency was significantly lower in the BiPAP group compared to control group consistent with our results (22). In addition, Surfactant therapy was reported with or without NCPAP.

In a study by Rojas et al., premature neonates with RDS were divided into two groups who received surfactant with or without NCPAP. They finally reported that MV dependency decreased from $39 \%$ in neonates treated with NCPAP to $26 \%$ in neonates treated by early surfactant, which is deferent with our study protocol (23). Gopel et al. prescribed surfactant in addition to CPAP in preterm neonates with spontaneous breathing and RDS. They found that these patients had a decreased rate of MV dependency in comparison with control group, which is similar to our findings (24).

In the present study, repeated administration of surfactant versus single dose was not effective enough to change the rate of INSURE failure. In contrary, Dani et al. demonstrated that multiple INSURE strategy could increase the success rate of INSURE and might prevent MV dependency, but the method of this study was different with our one in case selection which can justify these differences $(7,25)$. Rate of INSURE failure in the present study was $35.6 \%$ similar to other studies (26). Arterial pressure of carbon dioxide, mean arterial-to-alveolar oxygen pressure ratio and severe radiological abnormalities were previously reported as risk factors for INSURE failure which are not coherent with the results of our study (26). In this study and after adjusting confounding variables, birth weight was found as the only risk factor for INSURE failure which is consistent with Dani et al.'s study results (25). In addition, Cherif et al. demonstrated that the mortality rate was significantly higher among infants with INSURE failure and our study findings confirmed this result (26).

MV dependency (or Need to long time MV) is one of the dangerous complications of MV among infants with RDS. Transient intubation and CPAP can somewhat reduce this problem. Dani et al. showed that neonates treated with transient intubation and NCPAP rather than neonates treated with continuous MV had higher rate of MV dependency and this finding is concordant with our result (19). Additionally, in the present study, the rate of oxygen therapy dependency in INSURE group was less than the rate in MV group and this finding is similar to previous results (20).

On the other hand, previous reports evaluated the time of surfactant prescription (early or late) and some of them showed that the early surfactant therapy is not always effective enough to prevent MV dependency (27). Escobedo et al. showed that the duration of hospitalization and adverse outcomes of preterm neonates with RDS treated with early intubation and surfactant administration were not significantly different with patients treated with routine protocol (20). In a systematic review, Stevens et al. concluded that the frequency of MV dependency, BPD and air leak syndrome in a group of neonates treated with early surfactant therapy and NCPAP were less than a group treated with delayed surfactant therapy and MV, and this finding is similar to our findings (8).

We also found that birth weight, rate of ROP, delivery via C/S and presence of lung bleeding could be associated with neonatal mortality seen in the MV group and is concordant with previous reports $(28,29)$. CPAP can enhance the frequency of hospitalization complications such as NEC which is not in agreement with our results (30).

There were some limitations in this study; first, our sampling method was consecutive method and our allocation method was simple allocation (using even and odd numbers). We had to use the most available methods for randomization and allocation. Second, we did not follow these cases for long time. Third, the follow-up duration should be more similar in both groups. There were some confounding factors such as age and Apgar score, which were neutralized by logistic regression models.

The present study conducted to reply this important question that; "whether early administration of surfactant followed by quick extubation and NCPAP is better than surfactant therapy followed by continues MV". Our findings on preterm VLBW infants with RDS declared that INSURE method is related to lower need to supplementary $\mathrm{O}_{2}$, mortality and the rate of pneumothorax compared to the routine strategy (surfactant therapy followed by $\mathrm{MV}$ ). In addition, we concluded that BW is a valuable predictor for efficacy of treatment regardless of treatment methods in VLBW neonates with RDS.

\section{Acknowledgements}

Authors would like to thank parents of patients who kindly cooperated in this study. Moreover, we acknowl- 
edge Dr. Ali Amini-Harandi for editing the manuscript.

\section{Authors' Contribution}

All authors were contributed equally in writing and editing of the manuscript.

\section{Financial Disclosure}

There was no conflict of interest to be declared by the authors.

\section{Funding/Support}

There was no funding or support.

\section{References}

1. Miller RJ, Sullivan MC, Hawes K, Marks AK. The effects of perinatal morbidity and environmental factors on health status of preterm children at age 12. JPediatr Nurs. 2009;24(2):101-14.

2. Patton CD, Schulman ES. Surfactant: clinical applications. Am Fam Physician. 1992;46(1):233-6.

3. Been JV, Zimmermann LJ. What's new in surfactant? A clinical view on recent developments in neonatology and paediatrics. Eur JPediatr. 2007:166(9):889-99.

4. Wyllie JP. Neonatal endotracheal intubation. Arch Dis Child Educ Pract Ed. 2008;93(2):44-9.

5. Apisarnthanarak A, Holzmann-Pazgal G, Hamvas A, Olsen MA, Fraser VJ. Ventilator-associated pneumonia in extremely preterm neonates in a neonatal intensive care unit: characteristics, risk factors, and outcomes. Pediatrics. 2003;112(6 Pt 1):1283-9.

6. Ratcliff JD. Home health admission and care of a pediatric ventilator-dependent client. Home Healthc Nurse. 2007;25(1):34-40.

7. Dani C, Corsini I, Bertini G, Pratesi S, Barp J, Rubaltelli FF. Effect of multiple INSURE procedures in extremely preterm infants. J Matern Fetal Neonatal Med. 2011;24(12):1427-31.

8. Stevens TP, Harrington EW, Blennow M, Soll RF. Early surfactant administration with brief ventilation vs. selective surfactant and continued mechanical ventilation for preterm infants with or at risk for respiratory distress syndrome. Cochrane Database Syst Rev. 2007(4):CD003063.

9. Morley CJ, Davis PG. Continuous positive airway pressure: scientific and clinical rationale. Curr Opin Pediatr. 2008;20(2):119-24.

10. te Pas AB, Davis PG, Kamlin CO, Dawson J, O'Donnell CP, Morley CJ. Spontaneous breathing patterns of very preterm infants treated with continuous positive airway pressure at birth. Pediatr Res. 2008;64(3):281-5.

11. Stevens TP, Blennow M, Soll RF. Early surfactant administration with brief ventilation vs selective surfactant and continued mechanical ventilation for preterm infants with or at risk for RDS. Cochrane Database Syst Rev. 2002(2):CD003063.

12. Murray PG, Stewart MJ. Use of nasal continuous positive airway pressure during retrieval of neonates with acute respiratory distress. Pediatrics. 2008;121(4):e754-8.

13. Morley CJ, Davis PG, Doyle LW, Brion LP, Hascoet JM, Carlin JB. Nasal CPAP or intubation at birth for very preterm infants. $N$ Engl J Med. 2008;358(7):700-8.

14. Sweet DG, Carnielli V, Greisen G, Hallman M, Ozek E, Plavka R, et al. European consensus guidelines on the management of neonatal respiratory distress syndrome in preterm infants - 2010 update. Neonatology. 2010;97(4):402-17.

15. Vidyasagar D, Narang A. Perinatal and neonatal care in developing countries. In: Fanaroff editor. neonatal and perinatal medicine. 9 ed: Elsevier Mosby; 2010. p. 124.

16. Bahman-Bijari B, Malekiyan A, Niknafs P, Baneshi MR. BubbleCPAP vs. Ventilatory-CPAP in Preterm Infants with Respiratory Distress. Iranian journal of pediatrics. 2011;21(2):151-8.

17. Thomson MA. Continuous positive airway pressure and surfactant; combined data from animal experiments and clinical trials. Biol Neonate. 2002;81 Suppl 1:16-9.

18. Lundstrom KE. Early nasal continuous positive airway pressure for preterm neonates: the need for randomized trials. Acta Paediatr. 2003;92(10):1124-6.

19. Dani C, Bertini G, Pezzati M, Cecchi A, Caviglioli C, Rubaltelli FF. Early extubation and nasal continuous positive airway pressure after surfactant treatment for respiratory distress syndrome among preterm infants $<30$ weeks' gestation. Pediatrics. 2004:113(6):e560-3.

20. Escobedo MB, Gunkel JH, Kennedy KA, Shattuck KE, Sanchez PJ, Seidner S, et al. Early surfactant for neonates with mild to moderate respiratory distress syndrome: a multicenter, randomized trial.J Pediatr. 2004;144(6):804-8

21. Sandri F, Ancora G, Lanzoni A, Tagliabue P, Colnaghi M, Ventura ML, et al. Prophylactic nasal continuous positive airways pressure in newborns of 28-31 weeks gestation: multicentre randomised controlled clinical trial. Arch Dis Child Fetal Neonatal Ed. 2004;89(5):F394-8.

22. Ancora G, Maranella E, Grandi S, Pierantoni L, Guglielmi M, Faldella G. Role of bilevel positive airway pressure in the management of preterm newborns who have received surfactant. Acta Paediatr. 2010;99(12):1807-11.

23. Rojas MA, Lozano JM, Rojas MX, Laughon M, Bose CL, Rondon $\mathrm{MA}$, et al. Very early surfactant without mandatory ventilation in premature infants treated with early continuous positive airway pressure: a randomized, controlled trial. Pediatrics. 2009;123(1):137-42.

24. Gopel W, Kribs A, Ziegler A, Laux R, Hoehn T, Wieg C, et al. Avoidance of mechanical ventilation by surfactant treatment of spontaneously breathing preterm infants (AMV): an open-label, randomised, controlled trial. Lancet. 2011;378(9803):1627-34.

25. Dani C, Berti E, Barp J. Risk factors for INSURE failure in preterm infants. Minerva Pediatr. 2010;62(3 Suppl 1):19-20.

26. Cherif A, Hachani C, Khrouf N. Risk factors of the failure of surfactant treatment by transient intubation during nasal continuous positive airway pressure in preterm infants. Am J Perinatol. 2008;25(10):647-52.

27. Bohlin K, Gudmundsdottir T, Katz-Salamon M, Jonsson B, Blennow $M$. Implementation of surfactant treatment during continuous positive airway pressure. J Perinatol. 2007;27(7):422-7.

28. Giapros V, Drougia A, Asproudis I, Theocharis P, Andronikou S. Low gestational age and chronic lung disease are synergistic risk factors for retinopathy of prematurity. Early Hum Dev. 2011;87(10):653-7.

29. Abrishami M, Maemori G, Boskabadi H, Yaeghobi Z, Mafi-Nejad S. Incidence and Risk Factors of Retinopathy of Prematurity in Mashhad, Northeast Iran. Iran Red Crescent Med J. 2013;15(3):229-33.

30. Aly H, Massaro AN, Hammad TA, Narang S, Essers J. Early nasal continuous positive airway pressure and necrotizing enterocolitis in preterm infants. Pediatrics. 2009;124(1):205-10. 